\title{
Does competition really drive population distributions?
}

Jennifer A. Gill, School of Biological Sciences, University of East Anglia, Norwich Research Park, Norwich, NR4 7TJ, UK

What are the processes that influence the distribution of animals? This has always been a central question in ecology, but it is a critically important question in conservation biology when we attempt to predict how distributions of animals will change in response to the human activities that are altering the availability of the resources on which animals depend.

Ecology has long focussed on competition for resources as a key driver of the distribution of populations, and important theoretical frameworks, such as Ideal Free Distribution (IFD) theory, have been developed to describe associations between resource distribution and animal distribution (Fretwell \& Lucas 1970, Sutherland 1996). IFD theory describes a process by which the distribution of animals across resource patches could result from individual strategies of maximising fitness by minimising resource competition, when individuals are 'free' to move unhindered between resource patches and have perfect knowledge of the quality of all patches allowing them to choose the 'ideal' patch. Competition among individuals for the available resources results in individuals selecting the patch in which their fitness is maximised, given the distribution of resources and of other individuals also exploiting those resources.

Concepts such as IFD have proven exceptionally useful frameworks for considering drivers of distribution patterns, but might this focus on competition for resources have constrained our exploration of other potential drivers of distributions? Could aggregation of individuals in areas with more abundant resources arise through processes other than competition for resources? As IFD theory illustrates, competition requires knowledge of the resource conditions in different patches, in order to facilitate choice of 'ideal' patches. Knowledge of spatial variation in resource distribution could be gained by individuals sampling different patches, but over what spatial scales is such sampling likely to be feasible? Resource availability can also vary temporally, particularly in seasonal environments and in areas with rapid resource depletion, and strong temporal variation in resource availability could quickly make patch knowledge out of date, and therefore redundant.

Gaining sufficient knowledge of resource availability to facilitate fitness maximisation would therefore likely require frequent sampling of patches over spatial and temporal scales sufficient to capture the variation in resource and competitor abundance. Consequently, a key question is at what scales individual animals sample their environments. For birds, the rapid increase in studies in which individuals are tracked is helping us to answer this question, and studies to date suggest that (a) individuals typically use a small fraction of the sites occupied by populations, (b) those sites are often used continuously for long periods of the annual cycle and (c) the same sites tend to be used consistently between years (Alerstam et al. 2006, van Wijk et al. 2016, Wellbrook et al. 2017, Gill et al. 2019, Tedeschi et al. 2019). These data would suggest that competition for resources could only influence distribution patterns at very local scales (ie within the areas experienced by individuals and the time-periods in which they are experienced).

If individuals are only able to assess spatial and temporal variation in resource availability over local scales, what other processes might explain larger-scale aggregations of individuals in areas with more resources (eg Quaintenne et al. 2011, Ydenberg et al. 2017)? Among birds, a key evolved capacity is philopatry; the ability to return to previously experienced locations. This capacity to return is likely to have been a major evolutionary driver of the extraordinary navigation skills that 
migratory species display (Winger et al. 2019). If individuals return to previously-experienced locations each year, then the patterns of aggregation that emerge across a range will be determined by the factors influencing which sites are encountered by new recruits to the population, and the survival rates of individuals using different sites. Aggregation of individuals in areas with more abundant resources could thus arise through a higher frequency of recruitment and/or higher levels of survival in those areas, and these patterns would then be maintained by life-long philopatry.

A key question to address is therefore what influences the probability of recruits encountering different sites across a range (Gill et al. 2019). For breeding sites, natal philopatry (returning to breed at your natal location) is common and, for many bird species, males often show stronger natal philopatry than females (Gunnarsson et al. 2011, Trochet et al 2016). Positive associations between local productivity and local breeding abundance could therefore potentially arise through the feedback loop of high local productivity leading to large numbers of natal recruits, and vice versa. Such associations could be exacerbated by the non-natally philopatric sex being more likely to recruit into larger populations (Gunnarsson et al. 2011, Morrison et al. 2016), which could occur because larger populations are more likely to be encountered (Gilroy et al. 2016) and/or because conspecific attraction cues are stronger at sites with more individuals (Danchin et al. 2004, Morrison et al. 2016). The simple presence of philopatry and variation in demography could therefore, in theory, produce aggregations of individuals in areas with high productivity without any need to invoke competition among individuals for the 'best' sites.

Recruitment into non-breeding sites could be a largely random process (ie the probability of recruitment into any site would be similar), in which case local population abundances would primarily be a function of local survival rates. However, recruitment into non-breeding sites could also be non-random, for example if recruits use social cues to inform their settlement through following conspecifics and/or being attracted to areas with conspecifics. Conspecific following or attraction could also generate a positive feedback loop, if the majority of experienced conspecifics are travelling to sites with larger local populations and are thus more likely to be encountered by recruits. Seasonal variation in timing of movement of conspecifics to non-breeding sites could also influence encounter rates with recruits, and thus their resulting settlement patterns. For example, early recruits might be more likely to encounter early-migrating adult conspecifics (eg individuals that have failed to breed successfully or come from breeding areas on earlier schedules), and these patterns may vary between years.

If distributions are driven by factors such as social cues and philopatry influencing recruitment and survival, then our predictions for how population distribution might alter in response to environmental change will need to be constructed around these processes. There are likely to be important differences between competition-driven distribution and social cue/philopatry-driven distribution. For example, as competition-driven distribution requires knowledge of alternative locations, models might predict quite rapid and directional responses to environmental changes, as individuals are more likely to be able to move to alternative suitable locations as a result of their sampling behaviour. By contrast, a social cue/philopatry-driven distribution is likely to respond more slowly, as generational change is required to alter distributions, and both philopatry and conspecific attraction will tend to constrain distributions, even if suitable alternatives become available. In competition-driven distributions, environmental change could therefore directly facilitate distribution changes while, in a social cue/philopatry-driven distribution, changes in settlement probabilities of recruits and/or survival rates may be needed to drive distribution changes, and changes in local population growth rates may have little to do with local resource availability or 
carrying capacities, but may instead reflect changes in the processes influencing recruit distribution (eg Méndez et al. 2018).

If processes such as social cues and philopatry turn out to be important drivers of distribution patterns, we might reasonably ask why our focus has remained so firmly on resource competition for so many decades? Perhaps our capacity to observe whole systems, in contrast to the limited experience of individuals within those systems, has led us to assume greater individual knowledge and 'choice' than is realistic? Perhaps competition for resources and territorial exclusion of others reflects the issues that we consider to be important in human societies, and we have inadvertently projected this view to other species. Given that the majority of ecologists have, until recent decades, been men, perhaps a masculine-focus on competition and territoriality has unduly dominated our thinking? Whatever the reason, resource competition driving site 'choice' has been a pervasive concept in ecology, but rarely do we consider how achievable the assumptions of these concepts are likely to be, or what other processes could be involved.

What data do we need in order to unravel the relative contributions of different drivers of distributions? Most importantly, understanding the range of sites experienced by individuals, and the periods of the annual cycle when they are occupied, will greatly help us to understand the range of options from which choice is feasible. To understand the contribution of recruitment and survival to distributions, we need to quantify the frequency and scale of philopatry, the circumstances that influence philopatry and dispersal, and the role that social cues (and lack of social cues) play in determining individual experiences. All of these questions require tracking of individuals, especially juveniles, and we are increasingly able to explore these important questions. Finally, other processes could also be involved in determining individual and population-level distribution, and I hope that this perspective will encourage further thinking, exploration and testing of ideas, free from the constraints of previous assumptions.

\section{Acknowledgements}

I am indebted to José Alves, Graham Appleton, James Gilroy, Tómas Gunnarsson, Becky Laidlaw, Verónica Méndez and Cat Morrison for comments on this manuscript and for the many discussions from which these ideas developed.

\section{References}

Alerstam, T., Hake, M. \& Kjellén, N. (2006) Temporal and spatial patterns of repeated migratory journeys by ospreys. Animal Behaviour, 71, 555-566.

Danchin, E., Giraldeau, L. A., Valone, T. J. \& Wagner, R. H. (2004) Public information: from nosy neighbors to cultural evolution. Science, 305, 487-491.

Fretwell S.D. \& Lucas H.L. (1970) On territorial behaviour and other factors influencing habitat distribution in birds. Acta Biotheoretica 19, 16-36

Gill, J. A., Alves, J. A. \& Gunnarsson, T. G. (2019) Mechanisms driving phenological and range change in migratory species. Philosophical Transactions of the Royal Society B, 374, 20180047.

Gilroy, J. J. \& Lockwood, J. L. (2016) Simple settlement decisions explain common dispersal patterns in territorial species. Journal of Animal Ecology, 85, 1182-1190. 
Gunnarsson, T. G., Sutherland, W. J., Alves, J. A., Potts, P. M. \& Gill, J. A. (2011) Rapid changes in phenotype distribution during range expansion in a migratory bird. Proceedings of the Royal Society B: Biological Sciences, 279, 411-416.

Quaintenne, G., van Gils, J. A., Bocher, P., Dekinga, A. \& Piersma, T. (2011) Scaling up ideals to freedom: are densities of red knots across western Europe consistent with ideal free distribution?. Proceedings of the Royal Society B: Biological Sciences, 278, 2728-2736.

Méndez, V., Gill, J. A., Alves, J. A., Burton, N. H. \& Davies, R. G. (2018) Consequences of population change for local abundance and site occupancy of wintering waterbirds. Diversity and Distributions, 24, 24-35.

Morrison, C. A., Robinson, R. A., Clark, J. A. \& Gill, J. A. (2016) Causes and consequences of spatial variation in sex ratios in a declining bird species. Journal of Animal Ecology, 85, 1298-1306.

Sutherland, W. J. (1996) From individual behaviour to population ecology. Oxford University Press.

Tedeschi, A., Sorrenti, M., Bottazzo, M., Spagnesi, M., Telletxea, I., Ibàñez, R., Tormen, N., De Pascalis, F., Guidolin, L. \& Rubolini, D. (2019) Interindividual variation and consistency of migratory behavior in the Eurasian woodcock. Current Zoology. https://doi.org/10.1093/cz/zoz038

Trochet, A., Courtois, E. A., Stevens, V. M., Baguette, M., Chaine, A., Schmeller, D. S, Clobert, J. \& Wiens, J. J. (2016) Evolution of sex-biased dispersal. The Quarterly Review of Biology, 91, 297-320.

van Wijk, R. E., Bauer, S. \& Schaub, M. (2016) Repeatability of individual migration routes, wintering sites, and timing in a long-distance migrant bird. Ecology and evolution, 6, 8679-8685.

Wellbrock, A. H. J., Bauch, C., Rozman, J. \& Witte, K. (2017) 'Same procedure as last year?' Repeatedly tracked swifts show individual consistency in migration pattern in successive years. Journal of Avian Biology, 48, 897-903.

Winger, B. M., Auteri, G. G., Pegan, T. M. \& Weeks, B. C. (2019) A long winter for the Red Queen: rethinking the evolution of seasonal migration. Biological Reviews, 94, 737-752.

Ydenberg, R. C., Barrett, J., Lank, D. B., Xu, C., \& Faber, M. (2017). The redistribution of non-breeding dunlins in response to the post-DDT recovery of falcons. Oecologia, 183(4), 1101-1110. 